\title{
La noche de la ciudad translúcida
}

The translucent city night

Shimrit Florsheim

\section{Filiación}

Alumna de la F.A.U. Universidad de Chile.

E-mail:shim_f@yahoo.com

\section{Resumen}

Se presenta la innovación del hormigón translúcido, analizando sus posibilidades en arquitectura y urbanismo, además de mostrar sus ventajas en beneficio del ahorro energético y calidad de vida de los habitantes.

\section{Palabras clave}

Hormigón translúcido, ciudad en la noche, Bill Price, Áron Losonczi, Shimrit Florsheim.

\begin{abstract}
The following article presents a creation never seen before: the invention of the translucent concrete. This article analyzes its contribution to Architecture and Urbanism of these days, considering its advantages related with the energy saving and the life quality of the citizens.
\end{abstract}

\section{Key words}

Translucent concrete, city at night, Bill Price, Áron Losonczi, Shimrit Florsheim.

\section{Sumario \\ Introducción \\ El nacimiento de un nuevo material \\ El hormigón y la fibra óptica \\ El hormigón translúcido y la ciudad \\ Referencia}

\section{Introducción}

Cierre los ojos, relaje su mente e imagine una ciudad llena de luces encendidas, personas circulando, ascensores que trabajan sin parar y automóviles que estacionan y vuelven a marchar... Imagine una ciudad en movimiento. Una ciudad honesta, donde la vida se refleja sin antifaz, donde las fachadas de los edificios se desligan de sus pieles y los muros opacos se vuelven ligeros y permiten el traspaso de la luz... Imagine una Ciudad Translúcida.

Esta imagen ya no es un sueño, porque hoy, a comienzos del año 2005, estamos más cerca que nunca de transformar esta visión en realidad. Hoy, estamos frente a una nueva Arquitectura que producirá a su vez un nuevo Urbanismo.

Este cambio es posible de lograr gracias a una innovación que rompió con uno de los esquemas más rígidos existentes hasta ahora: el hormigón translúcido, un hormigón 
que permite el traspaso de la luz y el reflejo de siluetas y sombras a través de él. Parece insólito, pero es real, existe y sus efectos estéticos y constructivos son asombrosos, permitiendo la creación de espacios acogedores, seguros e innovadores.

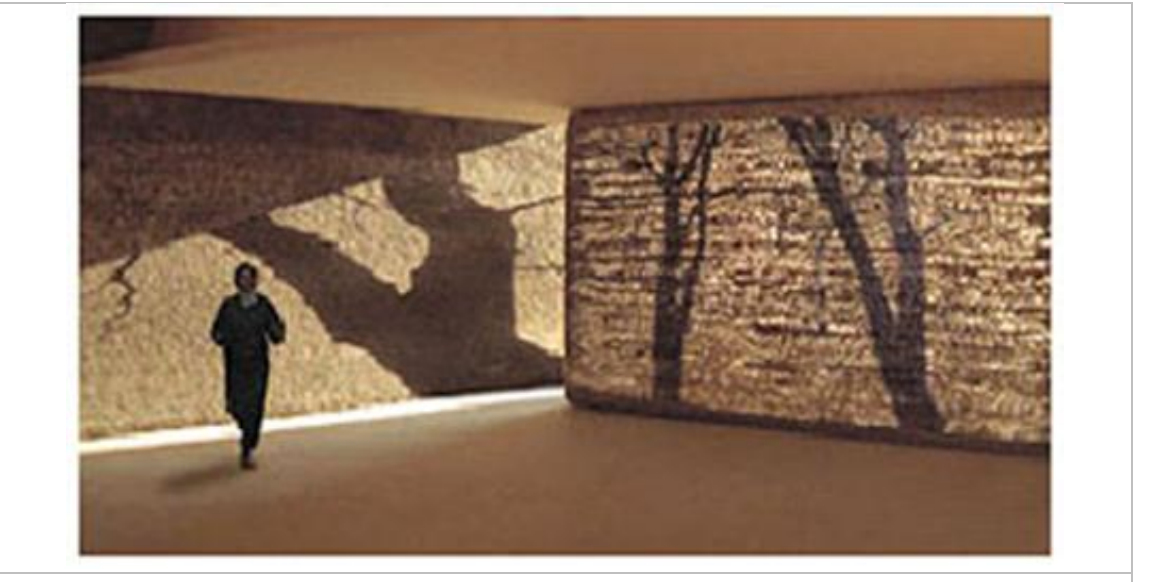

Fig.1. Efecto interior de aplicación en la arquitectura. Fte.: Internet.

La invención del hormigón translúcido podría cambiar de manera radical la imagen completa de las ciudades contemporáneas. Tal vez, luego del paso de los años, al detenernos y observar hacia atrás podamos apreciar que tuvimos la suerte de vivenciar una verdadera revolución...

Estos pensamientos vinieron a mi mente luego de leer un breve artículo que comentaba la aparición de este nuevo material. A pesar de que el artículo no proporcionaba mayores detalles, no pude dejar de pensar en el atractivo potencial que posee este invento sorprendente, al punto que decidí tomarlo como tema para mi Seminario de Investigación que debía realizar en el quinto año de la Carrera de Arquitectura, a pesar de todas las dificultades que podrían surgir al investigar un tema tan nuevo y reciente.

Junto con el apoyo de mi profesor guía ${ }^{1}$, comencé a navegar en un mar de signos de interrogación. ¿Cómo lograr transformar una noticia en un trabajo de investigación útil, y oportuno, especialmente para los arquitectos? Decidí, desde el comienzo, que ésa sería la meta que me guiaría.

Tomando en cuenta la escasa bibliografía que existe sobre el tema, el único camino que tuve para lograr recabar información sólida, confiable y más profunda era lograr contactarme con los propios creadores de este invento, lo que no fue fácil. Primero, y tal como haría un detective, comencé a buscar pistas que me guiaran hacia los lugares indicados y hacia las personas correctas.

Comunicándome con investigadores alrededor del mundo, fui derivada a periodistas y, por éstos, a profesores, los que, finalmente, me conectaron con arquitectos. Así pude llegar a las personas claves y empezar a unir diferentes pedazos de información

1 Arqto. Francis Pfenniger. 
adquiridos hasta armar la historia completa de la innovación y sus principales personajes. Y, justamente, gracias a la gentil disposición de uno de ellos, también conseguí una muestra de hormigón translúcido.

Debo confesar que al abrir la encomienda me sentí defraudada durante breves instantes, porque dentro del paquete había un ladrillo de hormigón común, sin ninguna apariencia especial. Pensé que tal vez, mi investigación de largos meses en realidad no tenía sentido, pero, al acercar el ladrillo hacia la luz, su apariencia cambió totalmente. El mismo bloque gris y opaco que tenía en mis manos cobró vida, se convirtió de pronto en un material luminoso y ligero. Ahí estaba el hormigón translúcido que había estado buscando. Se abrieron entonces en mi mente múltiples posibilidades de utilización de este material en la arquitectura y en el urbanismo.

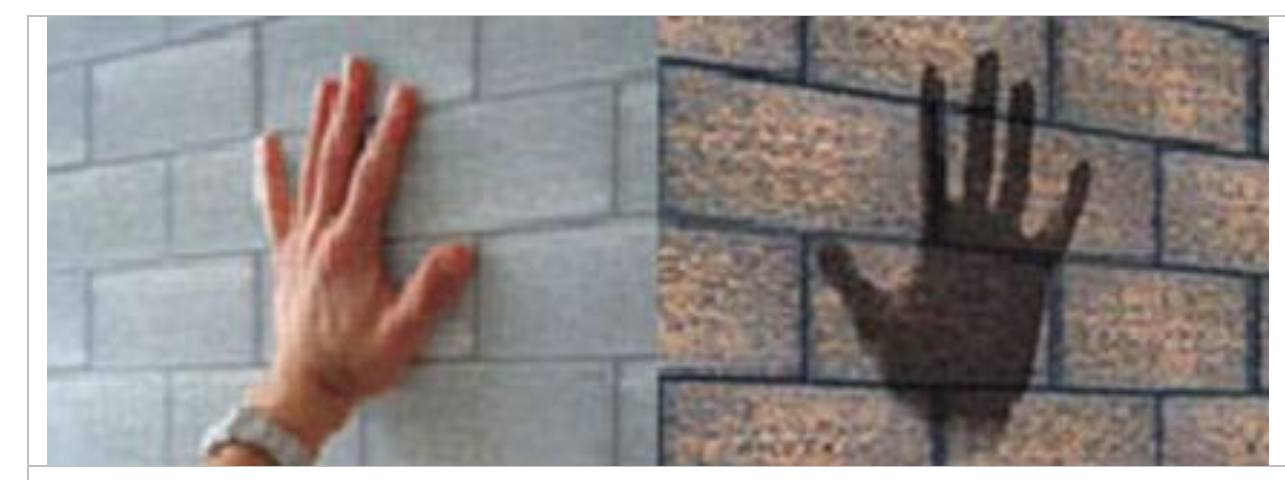

Fig. 2. Vista exterior e interior en aplicación. Fte.: Internet.

\section{EI nacimiento de un nuevo material}

La historia del hormigón translúcido comienza hace tan sólo seis años, cuando el arquitecto estadounidense Bill Price creó, en 1999, la primera muestra de este material.

Durante el trabajo en la oficina de Arquitectura OMA, junto a Rem Koolhaas, Bill Price comenzó a preguntarse sobre la translucidez relacionada con el hormigón, analizando la posibilidad de fabricar un hormigón que permitiera el paso de la luz.

La primera muestra realizada por Price fue el resultado de un estudio que trató de encontrar cuáles de los principales componentes del hormigón era posible sustituir para alcanzar la translucidez y, aun así, mantener su composición básica. De esta manera, el primer hormigón translúcido fue realizado a base de pedazos de vidrio y plástico translúcido.

Las fantásticas visiones de Bill Price lo llevaron a fabricar diferentes tipos de muestras de hormigón translúcido y aún hoy, durante estos días, Price sigue perfeccionando su invento a fin de adaptarlo a cualquier condición de uso. 
Muchos han tratado, y algunos lo han logrado, de alcanzar y concretar un hormigón con estas características, sin embargo, la real invención del hormigón translúcido se le atribuye a un joven arquitecto de 28 años de origen húngaro llamado Áron Losonczi.

\section{El hormigón y la fibra óptica}

Losonczi fue el único que logró patentar el producto en el año 2002 y crear su propia compañía. El hormigón creado por Losonczi el cual se lanzará al mercado durante los primeros meses de este año (2005), es un hormigón capaz de trasmitir la luz a través de él, gracias a fibras ópticas embutidas en su interior.

Las ventajas y las cualidades de la fibra óptica son asombrosas. Una de las más notorias es su capacidad de trasmitir la luz de un extremo a otro en cualquier condición, donde quiera que el filamento vaya - incluyendo curvas y esquinas - sin interrupción.

Esta es una condición sumamente importante en el momento de querer captar la luz y guiarla a un determinado lugar. Aquella particular característica puede ser la base de impresionantes y creativas aplicaciones.

No es casual, pues, que la fórmula que alcanzó el mayor éxito hasta ahora, por lograr transformar el hormigón en un material traslúcido y también mantener sus propiedades originales, es la que incorpora la fibra óptica a la mezcla del hormigón. Las características y propiedades del nuevo hormigón son prácticamente iguales al del tipo tradicional; su uso puede verse limitado sólo en el momento que se acabe la imaginación humana. Así, el nuevo hormigón tiene características nunca vistas hasta ahora y con un valor agregado indiscutible.

La nueva creación ha hecho posible cambiar dramáticamente la apariencia, la percepción, la sensación y la calidad de un determinado espacio, logrando efectos mágicos.

Con el hormigón translúcido, el sol parece estar ahora en nuestras manos, se ha capturado el sol convirtiéndolo en una parte integral del interior de la arquitectura. 


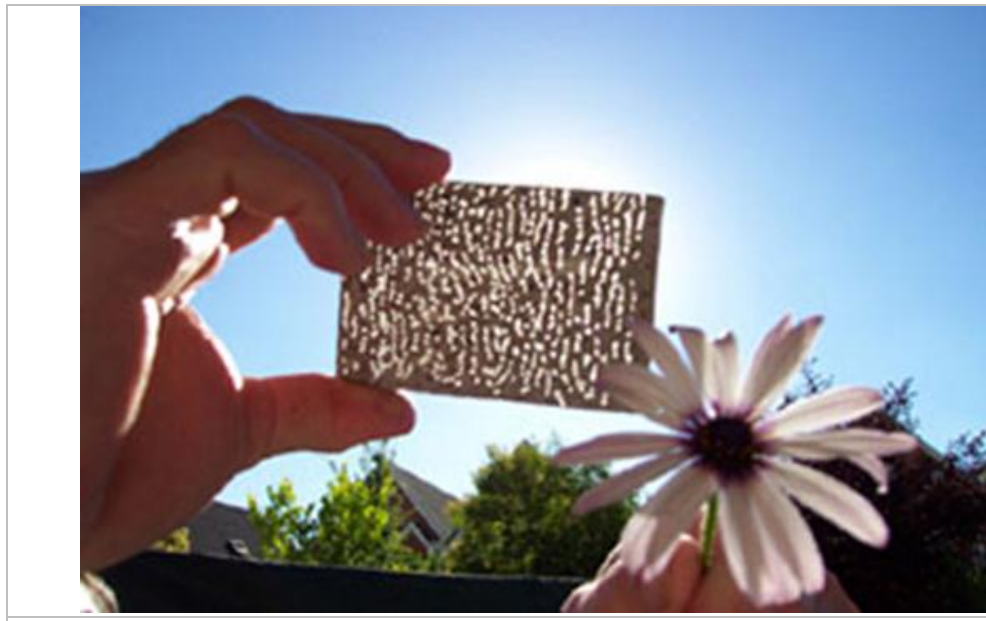

Fig. 3.- Foto de Shimrit Florsheim, a partir de la muestra obtenida. (C)

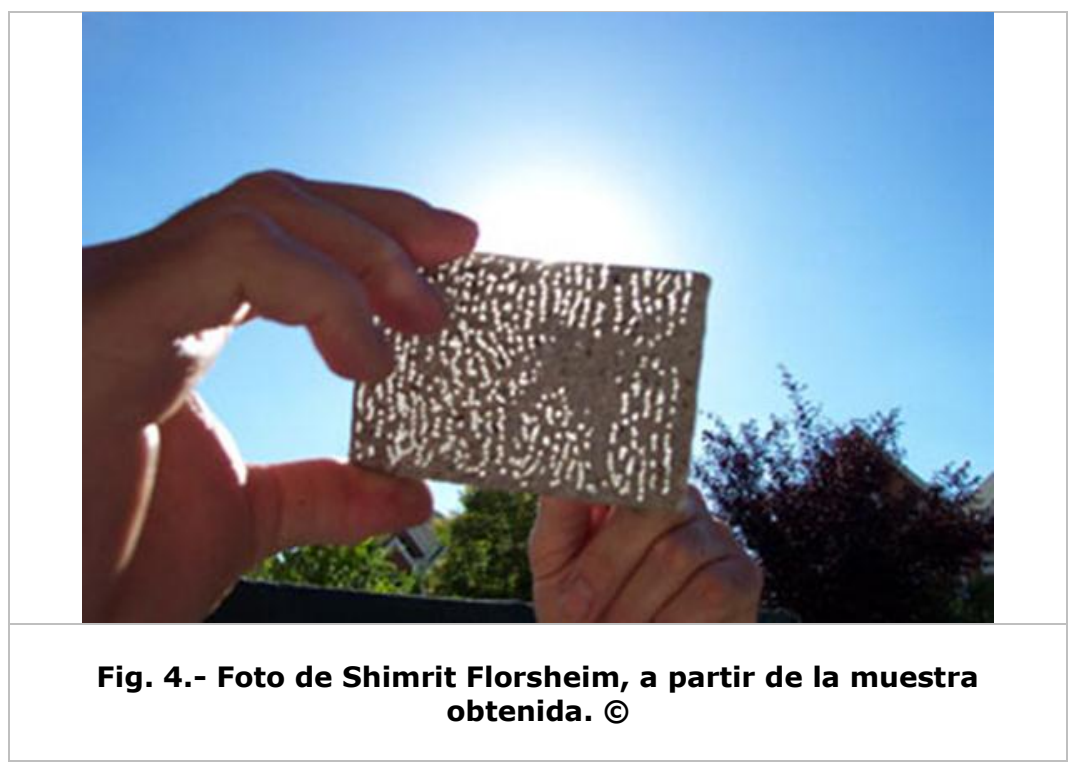

El efecto del nuevo material no es sólo estético. La posibilidad de transformar un muro tradicionalmente macizo y opaco en un muro translúcido que permite a la luz solar integrarse al espacio interior, agrega múltiples beneficios.

El sol es una fuente de energía, de libertad e iluminación. Estos factores tienen una alta influencia sobre los seres humanos, ya sea de manera consciente o inconsciente. La presencia de la luz natural aclara los espacios, descansa la vista, mejora el ánimo y beneficia la salud física y mental. Éste último no es un factor menor. Tal vez por eso en los países cálidos, donde el sol ilumina casi todo el año, pareciera ser que el nivel anímico y energético de los habitantes que allí viven es mucho más alto que en los países nublados en los cuales el sol casi nunca brilla. 
Una gran ventaja del hormigón translúcido es su capacidad de producir una luz tenue y suave sin necesidad de implementar accesorios adicionales como cortinas o persianas para reducir la intensidad de una luz que encandila. Se trata aquí de una "luz inteligente", una luz no invasiva, que otorga calidez a los espacios, además de establecer, de ahora en adelante, una nueva forma de interacción, constante, entre el interior y el exterior.

Se debe destacar, además, que al aprovechar mejor la luz solar es posible reducir notablemente el consumo de electricidad en los recintos, con el beneficio del ahorro energético que se logra.

Áron Losonczi ha exhibido su hormigón translúcido en diferentes lugares del mundo, especialmente en Europa, donde ha sido posible verlo hasta abril del 2005, en la exposición Ilamada "Liquid Stones", realizada en el National Building Museum, en Washington.

No es fácil cambiar la perspectiva de las personas acerca de un producto tan utilizado y tan popular como es el hormigón, que tiene una imagen estética única, característica y, que hasta ahora parecía intocable. Lograr introducir en la mente de las personas la nueva imagen del producto y, a la vez, ganar la confianza y apoyo, no es una tarea menor. Sin embargo, los encantos del Hormigón translúcido atrapan con sólo mirarlo.

A través de las exposiciones, Losonczi pudo demostrar las múltiples aplicaciones del nuevo producto, ya sea en el diseño como en la arquitectura y, de esta manera, ha logrado convencer y seducir hasta al más incrédulo. 


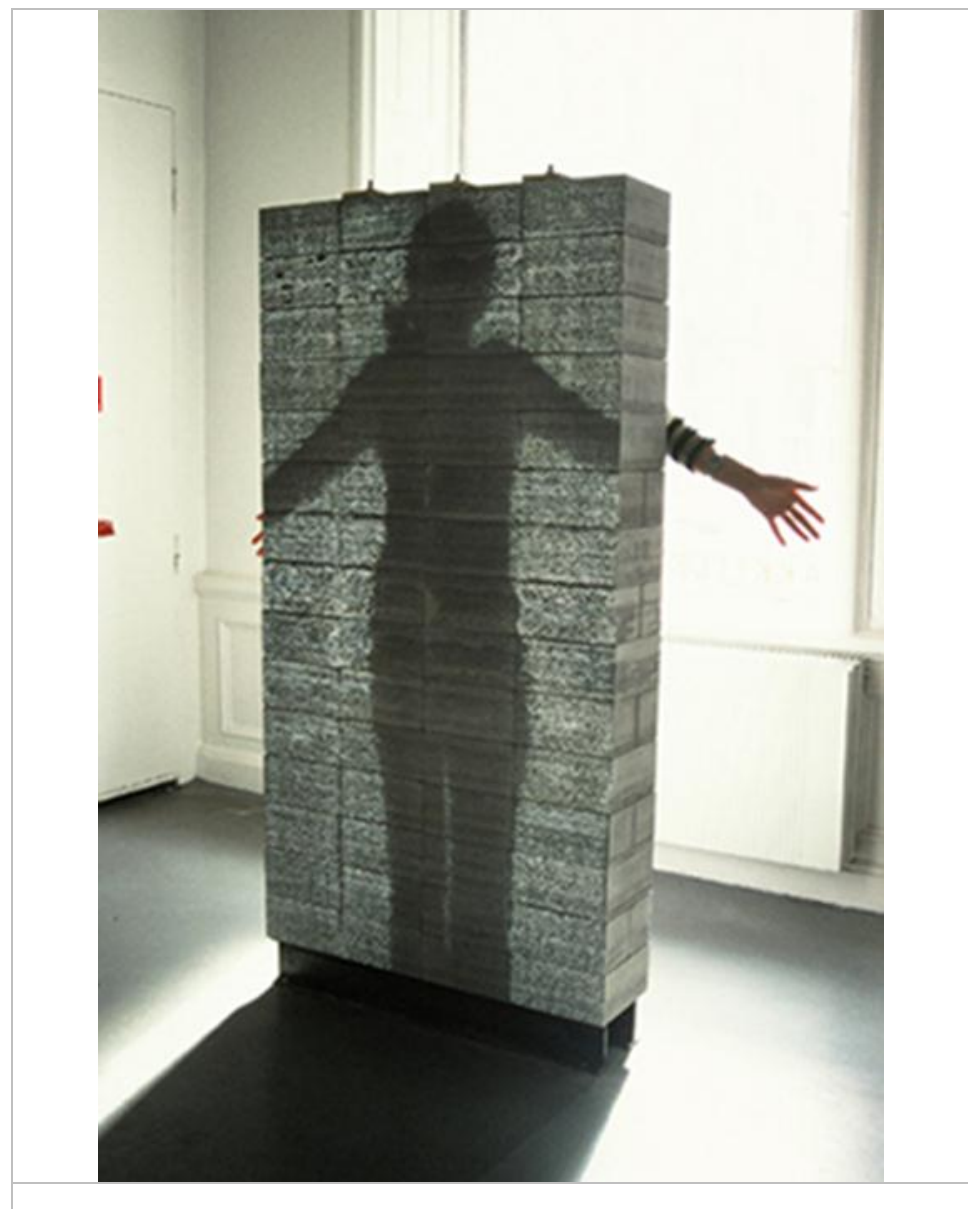

Fig. 5. Unidad demostrativa. Fte.: Áron Losonczi y Asociados. Gentileza a la autora.

Se han diseñado varios proyectos empleando el nuevo material, pero hasta el momento sólo un diseño se concretó. Éste es el 'LTC Lamp' - Lámpara diseñada en el año 2004. Esta lámpara quedará registrada en la historia como el primer objeto fabricado en Hormigón translúcido. Se han fabricado solamente 1.000 unidades de ella ${ }^{2}$.

2 Su costo actual [junio de 2005] por unidad es de 520 Euros. 


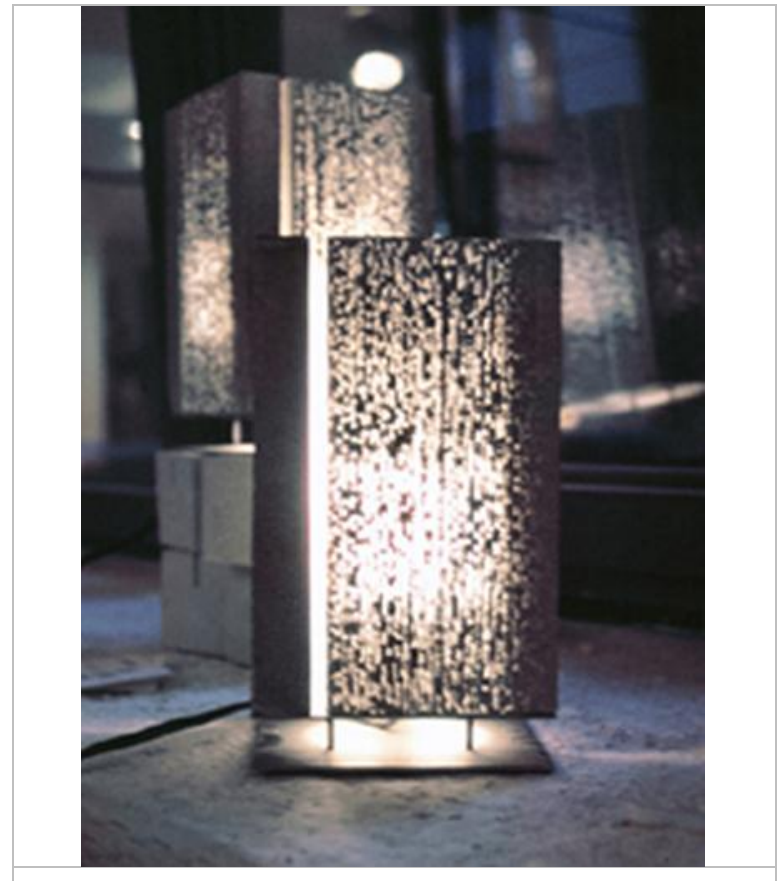

Fig. 6. Aplicación en lámpara. Fte.: Internet.

\section{El hormigón translúcido y la ciudad}

Si tomamos las cualidades básicas del objeto decorativo citado, ampliamos su escala, y lo llevamos a la Arquitectura, es también el Urbanismo de la ciudad el que dará un giro de 180 grados, porque al bajar el sol y caer la noche, la ciudad cobrará vida y con ella sus habitantes.

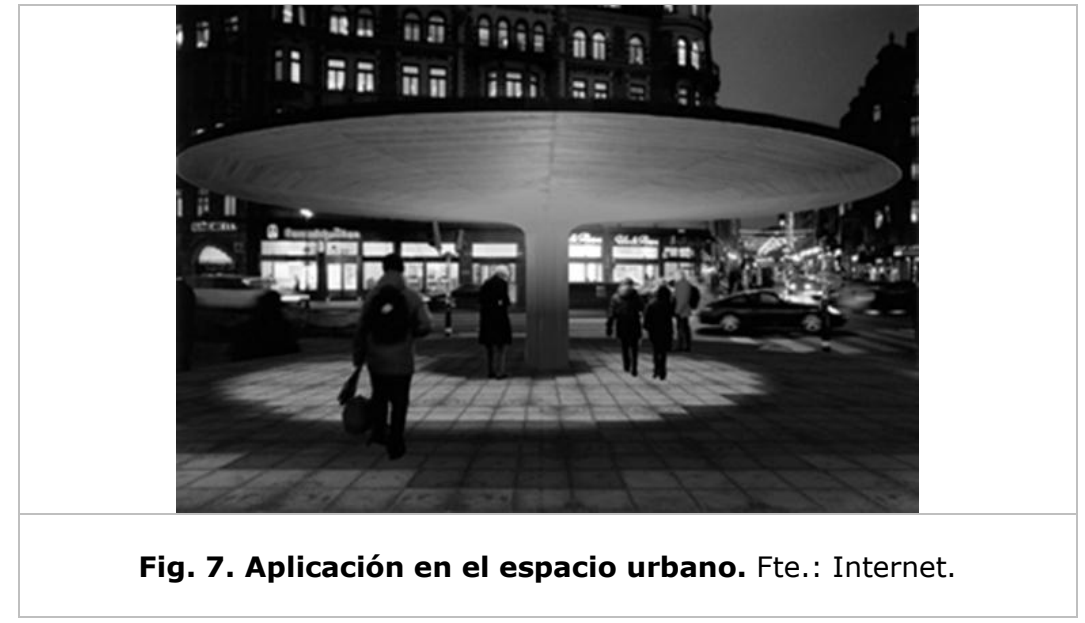


Un ejemplo de ello es el pavimento iluminado de una plaza pública de Estocolmo un proyecto realizado con hormigón translúcido, que refleja las singulares cualidades del nuevo hormigón y sus beneficios a la vida nocturna de la ciudad. Este proyecto fue diseñado especialmente para un concurso en el año 2002 y tuvo como objetivo realizar un juego de luz constante, mediante una plaza urbana interactiva que cambia de aspecto a cada instante.

Durante el día, los módulos cuadrados en el pavimento tienen la apariencia convencional del hormigón y la plaza parece ser una plaza urbana normal sin mayores atracciones.

Sin embargo, al caer la tarde, el lugar comienza a cambiar. Paulatinamente, la superficie de los módulos en el pavimento comienza a iluminarse, al dejar pasar la luz proveniente de la iluminación que se encuentra por debajo de ellos. De esta manera, al llegar la noche, la plaza llama la atención por su especial atractivo - múltiples bloques de $35 \times 35 \times 5 \mathrm{~cm}$. iluminados en el suelo invitan a recorrer el espacio, convirtiendo la plaza en un lugar iluminado, entretenido, vivo y seguro, sugiriendo además una cierta animación en los espacios públicos de la ciudad.

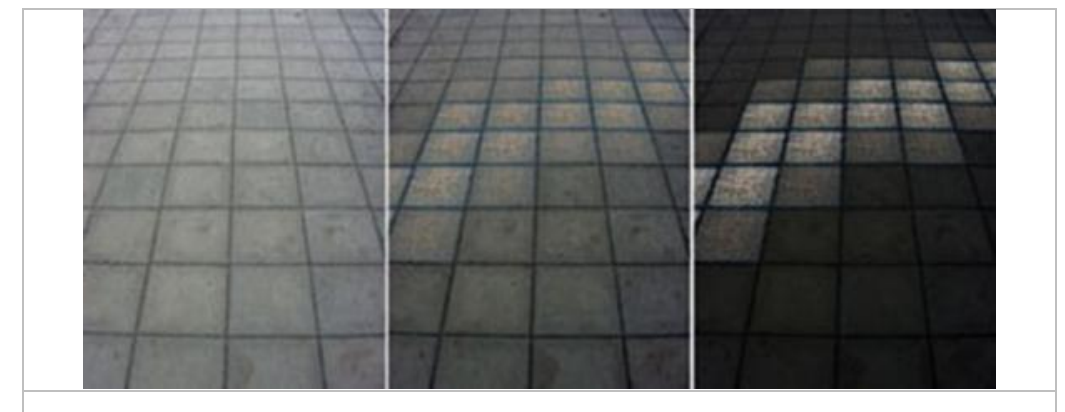

Fig. 8. Aplicación en el suelo. Fte.: Internet.

La sensación de seguridad es una de las características notables que aporta el nuevo material. Varios profesionales vinculados con este invento rescatan esta cualidad y algunos han llegado a denominarlo un material 'salvavidas', ya que su aporte a la arquitectura en ese sentido, puede llegar a ser decisivo. A raíz de esto, se han propuesto diferentes ideas para su empleo. Es así como, por ejemplo, se propone emplear el hormigón translúcido en los sectores de escape o salidas de emergencia, donde puede ser muy deseable que la luz se inserte desde el exterior, como cuando se produce un apagón producto de un temblor, de un incendio o corte de electricidad general en el edificio. En diálogos sostenidos con el Arqto. Sergio Amunátegui, este propuso su empleo en cárceles y en centrales eléctricas, donde, sin duda, sería de gran utilidad.

También sería muy útil su utilización en el caso de las estaciones de Metro y estacionamientos subterráneos para mejorar la calidad espacial de estos lugares "underground", reforzando el contacto entre el nivel de suelo y el nivel subterráneo. Se otorgaría, así, mayor seguridad y, a la vez, se haría un ahorro considerable de electricidad. 
Otra idea relacionada con la seguridad es emplear el nuevo hormigón en las vías para realizar los saltos de relieve, más comúnmente llamados 'lomos de toro' ('speed bump'), iluminándolos por debajo para así poder destacarlos más, facilitando la visión especialmente de noche, y aportando una suerte de luminaria, más que un objeto de restricción, que contribuye a la animación de la ciudad y a la seguridad ciudadana.

Con plazas, calles, carreteras, edificios, estaciones subterráneas -todas iluminadas con las debidas intensidades, en el debido momento y lugar-, la noche de la ciudad cambiará totalmente. Si pensamos en las ilimitadas creaciones que son posibles de realizar con el hormigón translúcido, podemos deducir que la ciudad del futuro que emplee este nuevo hormigón, no solo tendrá un aspecto estético diferente, sino que el cambio puede traer consigo muchas otras virtudes que pueden llegar a ser hasta más importantes aún. Porque, ¿qué se compara con un buen sentir de los propios habitantes en su propia ciudad?

Una ciudad segura, acogedora y preocupada por la salud física y mental de sus habitantes, preocupada del buen vivir y sentir de los ciudadanos, es una ciudad que prospera, una ciudad que refuerza el importante sentido de pertenencia e identidad de sus habitantes y estimula el desarrollo de actividades y la evolución hacia un futuro mejor.

\section{Referencia}

FLORSHEIM, Shimrit. Hormigón translúcido. Capturando la luz. El fin de la era gris. Seminario de Investigación, V Año, Carrera de Arquitectura, Prof. Guía Arqto. Francis Pfenniger, Departamento de Ciencias de la Construcción, Facultad de Arquitectura y Urbanismo de la Universidad de Chile, enero de 2005, 280 páginas ilustradas, cuadros, gráficos, bibliografía, (calificación máxima). 